\title{
Viscosity Effect on the Brownian Relaxation based Detection for Immunoassay Applications
}

\author{
Kai Wu, Lina Yu, Xiqian Zheng, Yi Wang, Yinglong Feng, Liang Tu and Jian-Ping Wang \\ Department of Electrical and Computer Engineering, University of Minnesota, Minneapolis, MN 55455
}

\begin{abstract}
Magnetic nanoparticles (MNPs) coated with Protein-G have been a model system to be used in different antibodies binding study. It is highly desirable to use a substrate-free biosensing system to detect antibodies binding in real-time. In this paper, we developed and applied a MNPs and search-coils integrated detection system, which is not only sensitive to the hydrodynamic volume of MNPs but also sensitive to the environment of MNPs, such as viscosity and temperature of the solution. We demonstrated that the viscosity effect influenced the amplitudes and phases of the 3rd ( $\mathrm{fH} \pm 2 \mathrm{fL})$ and 5 th $(\mathrm{fH} \pm \mathbf{4 f L}$ ) harmonics for the mixed frequency testing scheme. The binding between antibodies and Protein-G on MNPs increased hydrodynamic volume of particles, as a result, it also changed the amplitudes and phases of harmonics, which are the object signals we need to analyze. We demonstrated that the viscosity of antibody solution is lower than that of MNP solution, and the antibody binding effect could be shielded by the viscosity effect to certain extent.
\end{abstract}

\section{INTRODUCTION}

Magnetic nanoparticles (MNPs) have been utilized in biomedical detection systems because of its adjustable and comparable size to different molecules. Many research groups have pursued biochip based detection systems by utilizing the magnetic characteristic of MNPs. Ultra high sensitivity has been achieved for the detection of different molecules including antibodies. However, its limit is also obvious, the necessity of the to-be-detected molecules to bind onto the pre-coated substrate. Recently, the integration of MNPs and search-coils for a biosensing system has been quickly developed and adopted as a potential candidate for future substrate-free biosensing system [1-9]. One unclear point for this technique is the effect of viscosity of the solution, which will be investigated in this paper. Our experiment in this paper is based on the frequency mixing at the nonlinear region in the magnetization curve of superparamagnetic nanoparticles. Two sinusoidal magnetic fields with distinct frequencies are applied simultaneously to the sample: one with low frequency $\mathrm{f}_{\mathrm{L}}(50 \mathrm{~Hz})$ but high amplitude $\mathrm{A}_{\mathrm{L}}(100 \mathrm{Oe})$, written as $A_{L} \cos \left(2 \pi f_{L} t\right)$, the other is high frequency $\mathrm{f}_{\mathrm{H}}(35 \mathrm{KHz})$ but low amplitude $\mathrm{A}_{\mathrm{H}}(10 \mathrm{Oe})$, written as $A_{H} \cos \left(2 \pi f_{H} t\right)$. This low frequency field drives MNPs into nonlinear region and the high frequency field is then applied to generate mixing-frequency signals, which work as marks of different MNPs [1,3,10]. The response signal that contains a linear combination $\mathrm{mf}_{\mathrm{L}}+\mathrm{nf}_{\mathrm{H}}$ is then collected by a pair of balanced search coils. These harmonics are highly specific to the nonlinearity of the magnetization curve of the particles $[1,3,10]$. The relaxation mechanism for superparamagnetic nanoparticles is a joint effect of Néel and Brownian relaxation. For $\mathrm{Fe}_{3} \mathrm{O}_{4}$ particles with diameter smaller than $20 \mathrm{~nm}$, Néel relaxation dominates, and Brownian relaxation starts to dominate when its diameter is larger than 20nm [1]. It was demonstrated that antibodies bind to Protein-G layer on MNPs in real time, which changes the hydrodynamic volume of MNPs, leading to the change of the harmonics [3]. However, addition of antibody solution also changes environment of MNPs, such as the viscosity. In this study, the viscosity effect is analyzed and compared experimentally, and the immunoassay experiment is validated by considering the interference of the viscosity effect. We show experimentally that addition of antibody solution reduces the viscosity of MNPs solution, which increases the amplitude and decreases the phase of harmonics. And the binding of antibodies to MNPs increases the hydrodynamic volume, which decreases the amplitude and increases the phase of harmonics. We demonstrated that the viscosity factor attenuates the aimed signal in immunoassay application, which should be considered carefully for future search-coil based immunoassay applications.

\section{METHOD}

\section{A. Néel relaxation and Brownian relaxation}

As we apply the sinusoidal field $\mathrm{H}$, the relaxation time $\tau$ governs a MNP's ability to follow changes in the applied field via two relaxation mechanisms, Néel and Brownian relaxation. Néel relaxation is the rotation of magnetization inside the magnetic core,

$$
\tau_{N}=\tau_{0} \exp \left(\frac{K V_{m}}{k_{B} T}\right)
$$

where the time constant $\tau_{0}=10^{-9} s, \mathrm{~K}$ is the anisotropy constant of MNP, $V_{m}$ is the magnetic core 
volume of MNP, $k_{B}$ is Boltzmann constant, $\mathrm{T}$ is absolute temperature in Kelvin.

Brownian relaxation is the physical rotation of the hydrodynamic volume of MNP,

$$
\tau_{B}=\frac{3 \eta V_{H}}{k_{B} T}
$$

where $\eta$ is the viscosity of MNP solution, $V_{H}$ is the hydrodynamic volume of MNP.

Total relaxation time $\tau$ is a linear combination of $\tau_{N}$ and $\tau_{B}$ :

$$
\tau=\frac{\tau_{N} \cdot \tau_{B}}{\tau_{N}+\tau_{B}}
$$

\section{B. Magnetization theory}

Under two sinusoidal fields, the total field is

$$
H=A_{L} \cos \left(2 \pi f_{L} t\right)+A_{H} \cos \left(2 \pi f_{H} t\right)
$$

Magnetization of MNPs can be approximated by the static Lagnevin function [9]:

$$
\frac{M}{M_{s}}=L\left(\frac{m_{0} \mu_{0} H}{k_{B} T}\right)
$$

where $M_{s}$ is the saturation magnetization of the MNP, and $\mathrm{M}$ is the magnetization of MNP at field $\mathrm{H}, m_{0}$ is the magnetic moment of one particle, $\mathrm{H}$ is the total field as shown in formula (4). Taylor Expansion near zero magnetization shows the major mixing frequency components are as follows:

$$
\begin{aligned}
\frac{M}{M_{s}} & =L\left(\frac{m_{0} \mu_{0} H}{k_{B} T}\right) \\
& =\frac{1}{3}\left(\frac{m_{0} \mu_{0}}{k_{B} T}\right) H-\frac{1}{45}\left(\frac{m_{0} \mu_{0}}{k_{B} T}\right)^{3} H^{3}+\frac{2}{945}\left(\frac{m_{0} \mu_{0}}{k_{B} T}\right)^{5} H^{5}+\ldots \\
& =\ldots+\left[-\frac{1}{60} A_{H} A_{L}^{2}\left(\frac{m_{0} \mu_{0}}{k_{B} T}\right)^{3}+\frac{1}{252} A_{H}^{3} A_{L}^{2}\left(\frac{m_{0} \mu_{0}}{k_{B} T}\right)^{5}\right. \\
& \left.+\frac{1}{378} A_{H} A_{L}^{4}\left(\frac{m_{0} \mu_{0}}{k_{B} T}\right)^{5}+\ldots\right] \cdot \cos \left[2 \pi\left(f_{H} \pm 2 f_{L}\right) t\right] \\
& +\left[\frac{1}{1512} A_{H} A_{L}^{4}\left(\frac{m_{0} \mu_{0}}{k_{B} T}\right)^{5}+\ldots\right] \cdot \cos \left[2 \pi\left(f_{H} \pm 4 f_{L}\right) t\right]+\ldots
\end{aligned}
$$

Assuming a constant phase lag $\phi$ and no rotational motion of the carrier medium, we can obtain the actual phase lag $\phi$ and the magnetization amplitude $M_{0}$ shown as follows [11]:

$$
\begin{aligned}
& \phi=\arctan (\omega \tau) \\
& M_{0}=M \cdot \cos (\phi)
\end{aligned}
$$

where $\omega$ is angular frequency, $\tau$ is total relaxation time.
According to Lenz law, the induced voltage in search coil is proportional to

$$
V \propto \frac{d M_{0}}{d t} \cdot v_{\text {voulumeofparticle }}
$$

Thus, the collected signal in search coil is specific to characteristics of MNPs, such as relaxation time. The 3 rd and 5th harmonics are among the best candidates to intuitively show these characteristics.

\section{Experimental setup}

We firstly investigate the viscosity effect on the collected signal. A high frequency of $35 \mathrm{KHz}, 100 \mathrm{Oe}$ field and a low frequency of $50 \mathrm{~Hz}, 10 \mathrm{Oe}$ field are applied simultaneously. Then we put 30uL MNP solution into the search-coil system (shown in Figure 1) and monitor the real-time phase and amplitude in 3rd harmonic (at $35.1 \mathrm{KHz}$ ) and $5^{\text {th }}$ (at $35.2 \mathrm{KHz}$ ) harmonic. After 50 80 seconds, we added four solutions with the same volume of $50 \mathrm{uL}$ but different viscosities listed in Table 1. The signals were collected in real-time after the addition of solution with different viscosity for 150 seconds. Also, we studied the antibody binding effect under the same applied field. We put 50uL MNP solution, and prepared and test six different antibody samples listed in Table 2. We collected the signals before and after adding the different antibody samples.

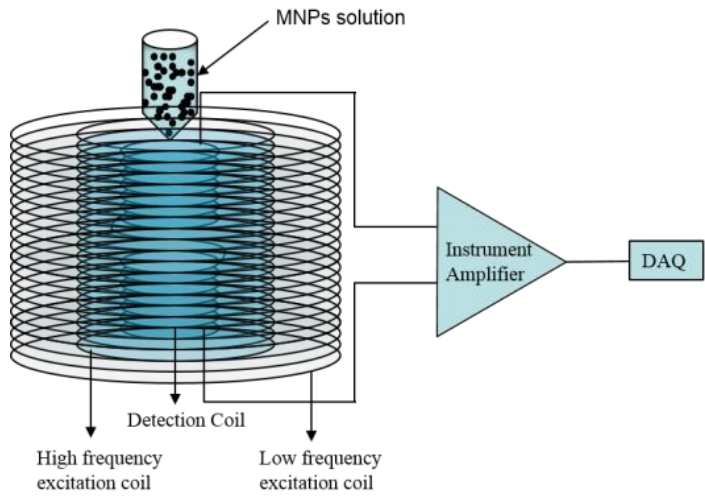

Figure 1. Search-coil based detection system.

\section{VISCOSITY EXPERIMENT}

In this paper, we use IPG-25 nanoparticles (purchased from Ocean NanoTech, iron oxide core MNPs of $25 \mathrm{~nm}$ diameter conjugated with around $10 \mathrm{~nm}$ Protein G layer, $1 \mathrm{mg} / \mathrm{mL}$ ). For nanoparticles in this size, Brownian relaxation dominates [1].

Table 1 Viscosity effect on the collected signals

\begin{tabular}{|c|c|c|c|c|}
\hline $\begin{array}{c}\text { Solution } \\
\text { (Room temperature) }\end{array}$ & $\begin{array}{c}\text { Amplitude of the } \\
\text { 3rd harmonic }\end{array}$ & $\begin{array}{c}\text { Phase of the 3rd } \\
\text { harmonic }\end{array}$ & $\begin{array}{c}\text { Amplitude of the } \\
\text { 5th harmonic }\end{array}$ & $\begin{array}{c}\text { Phase of the 5th } \\
\text { harmonic }\end{array}$ \\
\hline Water(0.95cp) & $+0.193 \mathrm{uV}$ & $-2.549^{\circ}$ & $+0.023 \mathrm{uV}$ & $+3.678^{\circ}$ \\
\hline PBS(unknown viscosity) & $+0.178 \mathrm{uV}$ & $-1.996^{\circ}$ & $+0.012 \mathrm{uV}$ & $+2.827^{\circ}$ \\
\hline $\begin{array}{c}25 \% \text { volume glycerol mixed with } \\
\text { water(viscosity 1.18cp) }\end{array}$ & $+0.144 \mathrm{uV}$ & $-0.924^{\circ}$ & $+0.041 \mathrm{uV}$ & $-0.285^{\circ}$ \\
\hline $\begin{array}{c}50 \% \text { volume glycerol mixed with } \\
\text { water(viscosity 1.59cp) }\end{array}$ & $-0.356 \mathrm{uV}$ & $+29.032^{\circ}$ & $-0.14 \mathrm{uV}$ & $+12.284^{\circ}$ \\
\hline
\end{tabular}

('+' indicates increase of amount, '-' indicates decrease of amount) 
According to formula (2) and (3), the relaxation time is expressed as $\tau \approx \tau_{B}=\frac{3 \eta V_{H}}{k_{B} T}$.If the viscosity of MNP solution decreases, then the relaxation time $\tau$ decreases, the phase lag $\phi$ decreases and the amplitude increases. If the viscosity increases, $\tau$ increases, the phase lag $\phi$ increases and the amplitude decreases. We added $50 \mathrm{uL}$ solutions with different viscosities into $30 \mathrm{uL}$ IPG-25 solution, respectively. Data are shown in table 1 and plotted in Fig. 2. Viscosities are calculated according to [12].

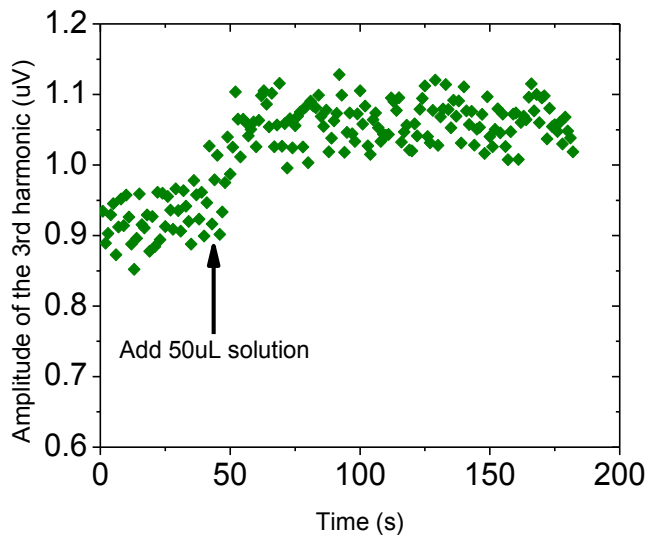

(a)

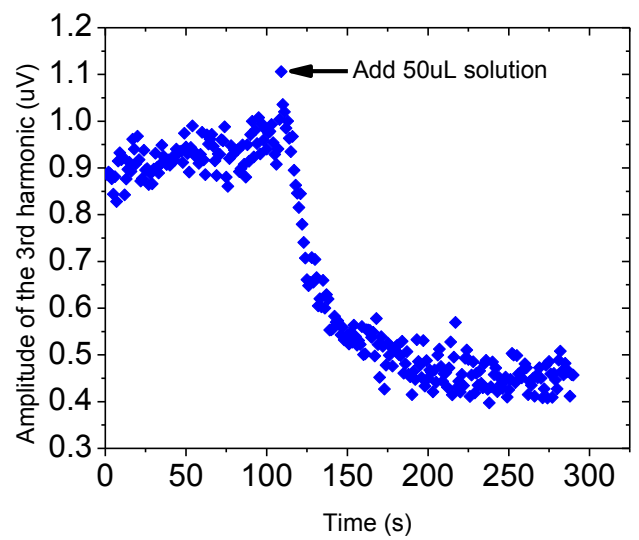

(b)

Figure 2. Viscosity effect on the amplitude of the 3rd harmonic (a) Add 25\%volume glycerol mixed with water; (b) Add 50\%volume glycerol mixed with water.

As shown in Table 1 and Fig 2, we can deduce that the viscosity of PBS is a little bit higher than water, it's between $0.95 \mathrm{cp}$ and $1.18 \mathrm{cp}$. And the viscosity of IPG-25 solution is between $1.18 \mathrm{cp}$ and $1.59 \mathrm{cp}$. The amplitude of the 5th harmonic increases or decreases as amplitude of the 3rd harmonic changes. The phase of the 5th harmonic may not change as the expected trend. That may be resulted from its relatively lower signal to noise ratio (SNR) compared to 3rd harmonic.

\section{IMMUNOASSAY EXPERIMENT}

Active antibodies can bind to Protein-G on MNPs, which increases the hydrodynamic volume of MNPs. According to formula (2) and (3), as the hydrodynamic volume of MNP increases, relaxation time $\tau$ increases, phase lag $\phi$ increases and the amplitude decreases. We used 50uL IPG-25 MNPs, added antibodies with different concentrations. Two control groups are tested with $50 \mathrm{uL}$ PBS solution and $50 \mathrm{uL}$ inactive antibody. Testing summary refers to Table 2.

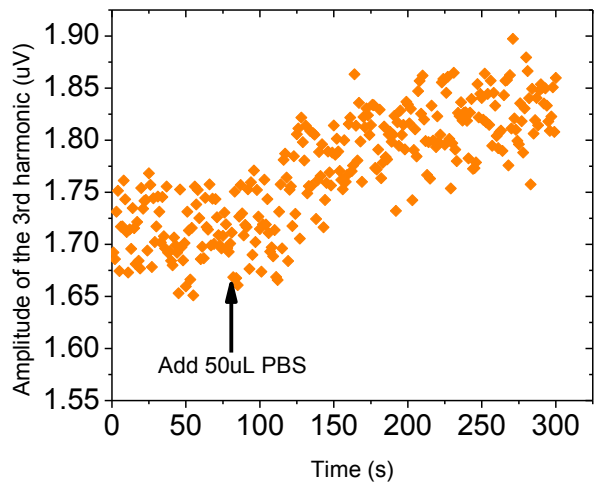

(a)

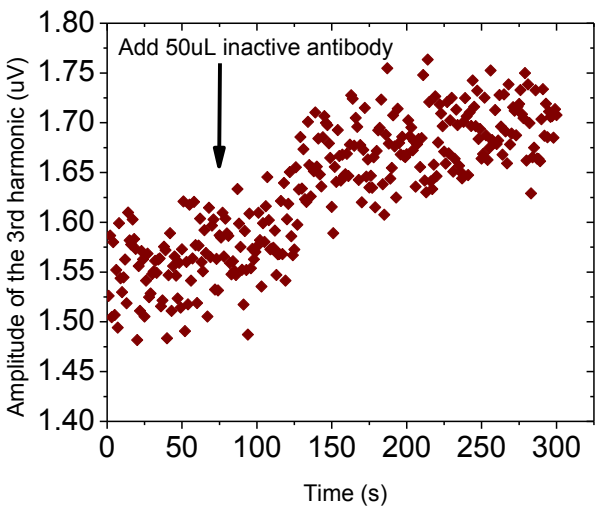

(b)

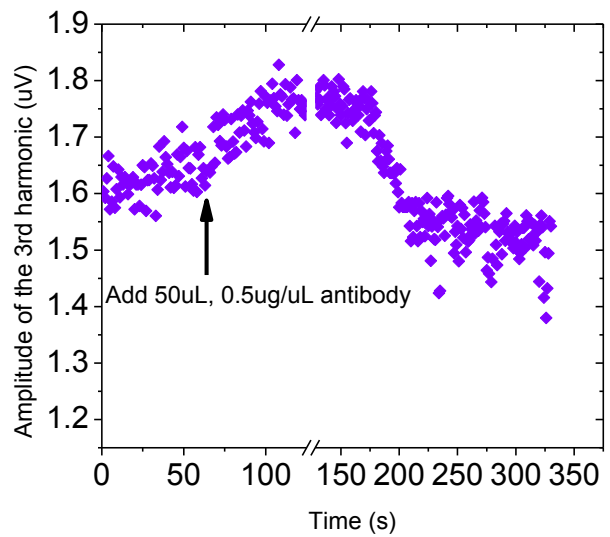

(c)

Figure 3. Antibody binding effect mixed with the viscosity effect on the amplitude of the 3rd harmonic (a) add 50uL PBS; (b) add $50 \mathrm{uL}$ inactive antibody; (c) add 50uL, $0.5 \mathrm{ug} / \mathrm{uL}$ antibody. 
Table 2 Antibody binding effect vs. viscosity effect

\begin{tabular}{|c|c|c|c|c|c|c|}
\hline Sample & 1 & 2 & 3 & 4 & 5 & 6 \\
\hline $\begin{array}{c}\text { Solution } \\
\text { (In room temperature) }\end{array}$ & $\begin{array}{c}\text { Antibody } \\
0.5 \mathrm{ug} / \mathrm{uL} 50 \mathrm{uL}\end{array}$ & $\begin{array}{c}\text { Antibody } \\
0.5 \mathrm{ug} / \mathrm{uL} 100 \mathrm{uL}\end{array}$ & $\begin{array}{c}\text { Antibody } \\
0.05 \mathrm{ug} / \mathrm{uL}, 50 \mathrm{uL}\end{array}$ & $\begin{array}{c}\text { Antibody } \\
0.025 \mathrm{ug} / \mathrm{uL}, 50 \mathrm{uL}\end{array}$ & $\begin{array}{c}\text { Inactive Antibody } \\
0.5 \mathrm{ug} / \mathrm{uL}, 50 \mathrm{uL}\end{array}$ & $\begin{array}{c}\text { PBS, } \\
50 \mathrm{uL}\end{array}$ \\
\hline Amplitude, 3rd harmonic & $-0.130 \mathrm{uV}$ & $-0.078 \mathrm{uV}$ & $-0.025 \mathrm{uV}$ & $+0.015 \mathrm{uV}$ & $+0.134 \mathrm{uV}$ & $+0.133 \mathrm{uV}$ \\
\hline Phase, 3rd harmonic & $+3.161^{\circ}$ & $+0.539^{\circ}$ & $+0.862^{\circ}$ & $-0.909^{\circ}$ & $-2.512^{\circ}$ & $-2.225^{\circ}$ \\
\hline
\end{tabular}

('+' indicates increase of amount, '-' indicates decrease of amount)

As shown in Table 2 and Fig 3, the viscosity of the antibody solution is close to that of PBS. From the collected data in sample 2, we added more antibody solution, and the antibody binding is expected to increase the average hydrodynamic volume in MNPs, thus the amplitude is supposed to reduce more than $0.13 \mathrm{uV}$ compared to sample 1 . However, we get a $0.078 \mathrm{uV}$ reduction in amplitude, which indicates that the addition of the antibody solution decreases the viscosity of MNP solution, thus increases the amplitude to some extent. In the antibody addition experiment, we added the antibody solution first, and found an immediate increase of the amplitude, then we waited for about 5 minutes after we saw a gentle drop in the collected signal, which indicates that upon the addition of antibody solution, the viscosity effect appears first, and after about 5 minutes' reaction, the antibody binding effect appears gradually.

\section{CONCLUSIONS}

In this paper, we first investigated the viscosity effect on the collected signals for a MNPs and search-coil integrated immunoassay detection system. We found that the addition of water, PBS, and $25 \%$ volume glycerol mixed with water (viscosity $1.18 \mathrm{cp}$ ) led to the increase of the amplitude and the decrease of the phase for the real-time signals. The Addition of 50\% volume glycerol mixed with water (viscosity $1.59 \mathrm{cp}$ ) led to the decrease of the amplitude and the increase of the phase. We could infer that the viscosity of MNP is between $1.18 \mathrm{cp}$ and $1.59 \mathrm{cp}$. The viscosity of human blood is $4 \sim 5 \mathrm{cp}$, so the addition of blood could lead to the decrease of the amplitude, and the effect of antibody binding could be easily sheltered by this viscosity effect. Secondly, we studied the antibody binding effect, when we add 50uL antibody $(0.5 \mathrm{ug} / \mathrm{uL})$ the amplitude decreased as we expected. But when adding $100 \mathrm{uL}$ antibody $(0.5 \mathrm{ug} / \mathrm{uL})$, the amplitude decreased less than that $50 \mathrm{uL}$ case. Furthermore, when we used $50 \mathrm{uL}$ antibody with a concentration of $0.025 \mathrm{ug} / \mathrm{uL}$, the amplitude was basically no change. This demonstrated in our previous experimental result that the viscosity of antibody solution is smaller than MNP solution, this will lead to increase of amplitude. But the antibody binding with MNPs could lead to the decrease of the amplitude. In summary we found that the binding effect and the viscosity effect work against each other. For future immunoassay applications, control experiments are even more critical and should be paid special attention.

\section{REFERENCES}

[1] Tu, Liang, Kai Wu, Todd Klein, and Jian-Ping Wang. "Magnetic nanoparticles colourization by a mixing-frequency method." Journal of Physics D: Applied Physics 47, no. 15 (2014): 155001.

[2] Lucchini, M. A., and F. Canepa. "Brownian relaxation of magnetic nanoparticles in fluid: the effect of the solvent." Journal of Nanoparticle Research 14, no. 4 (2012): 1-7.

[3] Tu, Liang, Ying Jing, Yuanpeng Li, and Jian-Ping Wang. "Realtime measurement of Brownian relaxation of magnetic nanoparticles by a mixing-frequency method." Applied Physics Letters 98, no. 21 (2011): 213702.

[4] Rauwerdink, Adam M., and John B. Weaver. "Measurement of molecular binding using the Brownian motion of magnetic nanoparticle probes." Applied Physics Letters 96, no. 3 (2010): 033702.

[5] Weaver, John B., Adam M. Rauwerdink, Charles R. Sullivan, and Ian Baker. "Frequency distribution of the nanoparticle magnetization in the presence of a static as well as a harmonic magnetic field." Medical physics 35, no. 5 (2008): 1988-1994.

[6] Nikitin, Petr I., Petr M. Vetoshko, and Tatiana I. Ksenevich. "New type of biosensor based on magnetic nanoparticle detection." Journal of Magnetism and Magnetic Materials 311, no. 1 (2007): 445-449.

[7] Hong, Chin-Yih, C. C. Wu, Y. C. Chiu, S. Y. Yang, H. E. Horng, and H. C. Yang. "Magnetic susceptibility reduction method for magnetically labeled immunoassay." Applied physics letters 88, no. 21 (2006): 212512.

[8] Chung, S-H., A. Hoffmann, K. Guslienko, S. D. Bader, C. Liu, B. Kay, L. Makowski, and L. Chen. "Biological sensing with magnetic nanoparticles using Brownian relaxation." Journal of applied physics 97, no. 10 (2005): 10R101.

[9] Chung, S. H., A. Hoffmann, S. D. Bader, C. Liu, B. Kay, L. Makowski, and L. Chen. "Biological sensors based on Brownian relaxation of magnetic nanoparticles." Applied physics letters 85, no. 14 (2004): 2971-2973.

[10] Krause, Hans-Joachim, Norbert Wolters, Yi Zhang, Andreas Offenhäusser, Peter Miethe, Martin HF Meyer, Markus Hartmann, and Michael Keusgen. "Magnetic particle detection by frequency mixing for immunoassay applications." Journal of Magnetism and Magnetic Materials 311, no. 1 (2007): 436-444.

[11] Dieckhoff, Jan, Meinhard Schilling, and Frank Ludwig. "Fluxgate based detection of magnetic nanoparticle dynamics in a rotating magnetic field." Applied Physics Letters 99, no. 11 (2011): 112501 .

[12] Cheng, Nian-Sheng. "Formula for the viscosity of a glycerolwater mixture."Industrial \& engineering chemistry research 47, no. 9 (2008): 3285-3288. 